\title{
A pathogenetic link between non-alcoholic fatty liver disease and celiac disease
}

\author{
Ludovico Abenavoli • Natasa Milic • \\ Antonino De Lorenzo • Francesco Luzza
}

Received: 27 April 2012/ Accepted: 13 June 2012

(C) Springer Science+Business Media, LLC 2012

\begin{abstract}
Non-alcoholic fatty liver disease (NAFLD) has recently been recognized as the leading cause of the abnormalities in the liver function tests in the Western countries. Celiac disease (CD) is a permanent immunological intolerance to gluten proteins in genetically predisposed individuals. CD has been reported in $4-13 \%$ of the cases with steatohepatitis, although the pathogenesis of the liver steatosis in CD patients is unclear. Based on the literature data, it can be concluded that the inclusion of serological markers of CD should be a part of the general workup in the patients with steatosis when other causes of the liver disease are excluded and in the patients with NAFLD when metabolic risk factors are not evident.
\end{abstract}

Keywords Non-alcoholic fatty liver disease $\cdot$ Celiac disease $\cdot$ Intestinal permeability $\cdot$ Microbiota

Non-alcoholic fatty liver disease (NAFLD) is an umbrella term that refers to a broad spectrum of lesions that range from isolated steatosis, to active lesions with inflammation (non-alcoholic steatohepatitis-NASH), to cirrhosis and hepatocellular carcinoma [1]. The prevalence of NAFLD in

L. Abenavoli $(\bowtie) \cdot$ F. Luzza

Department of Health Sciences, University "Magna Græcia",

Viale Europa, 88100 Catanzaro, Italy

e-mail:1.abenavoli@unicz.it

N. Milic

Department of Pharmacy, University of Novi Sad,

Hajduk Veljkova 3, 21000 Novi Sad, Serbia

A. De Lorenzo

Department of Neuroscience, Division of Human Nutrition,

University “Tor Vergata”, Via Montpellier n.1,

00133 Rome, Italy
Western countries, is about $30 \%$ [2, 3]. Celiac disease (CD) is an autoimmune disorder, which manifests in the permanent intolerance to gluten proteins in the genetically predisposed individuals [4]. These peptides are able to trigger two immunological pathways: one is thought to be a rapid effect on the epithelium of small-intestinal that involves the innate immune response, and the other represents the adaptive immune response involving $\mathrm{CD} 4^{+} \mathrm{T}$ cells in the lamina propria, that recognize gluten epitopes processed and presented by antigen presenting cells [5]. The prevalence is estimated to be $1 \%$ in European populations [6]. It has been reported that a gluten-dependent hepatic involvement is present in CD patients [7].

\section{The role of gut-liver axis}

The increased incidence of diabetes, obesity, hypertension, hypertriglyceridemia, and/or hypercholesterolemia in general population is considered to be the most common cause of NAFLD. A "two-hits" hypothesis of the NAFLD pathogenesis was proposed $[8,9]$. The first "hit" presents the insulin resistance leading to the fat accumulation resulting in steatosis. The second "hit" is an oxidative stress, determining lipid peroxidation and increasing cytokine production, that causes the inflammation and necrosis (Fig. 1). The human gastrointestinal tract is an ecosystem integrated by up to $10^{14}$ total bacteria, called microbiota [10]. Mucosal epithelium of the small intestine is the barrier between microbiota and gut lumen. The intestinal microflora is a primary source of the endotoxins (lipopolysaccharide-LPS) produced by Gram-negative bacteria, normally crossing the mucosa only in trace amounts, entering the portal blood and becoming cleared by liver [11]. The increased intestinal permeability appears 
Fig. 1 Possible pathogenetic link between NAFLD and CD

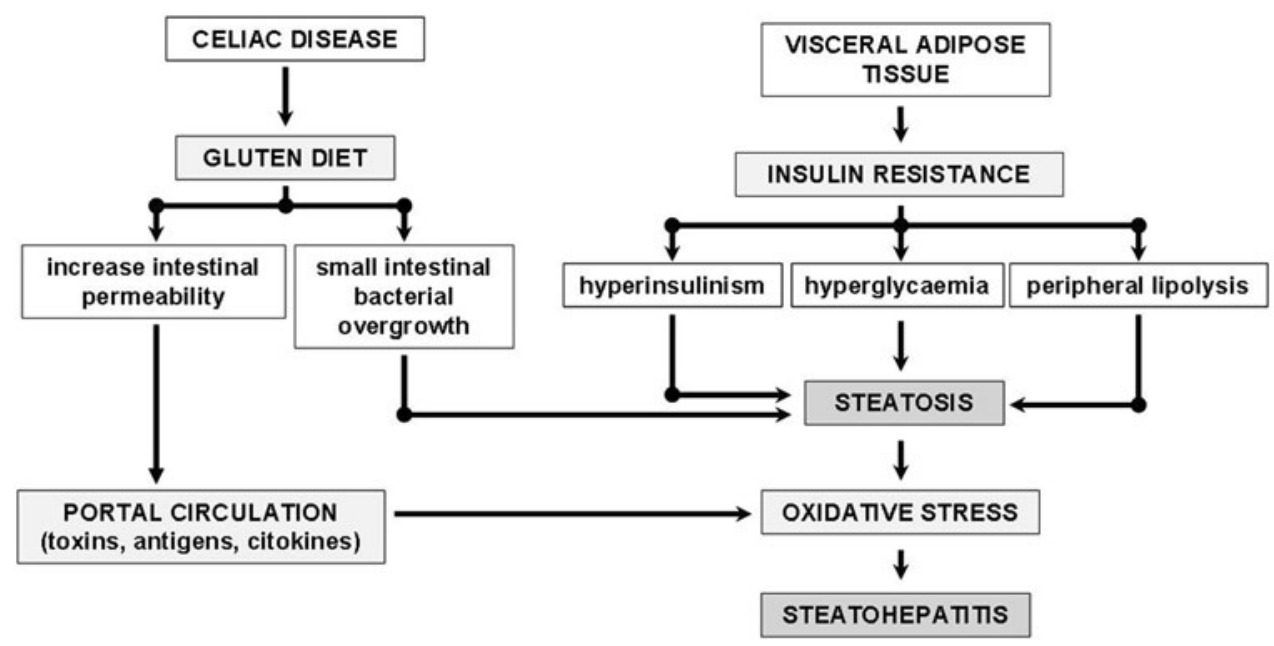

to be caused by disruption of the intercellular tight junctions. As a result of this, there are increased LPS and gutderived products such as peptidoglycan levels in portal circulations and increased levels of endotoxin-mediated cytokines in the liver (Fig. 1). LPS, as inflammatory factor, participates in the second "hit" and converts hepatic steatosis into steatohepatitis [12-14].

\section{Celiac disease and NAFLD}

The persistent elevation of transaminase levels is the most common liver abnormality found in untreated CD patients $[7,15]$. Increased permeability to intraluminal antigens could induce an immune response against antigens sharing common epitopes to self-liver proteins and/or against cryptic antigens unmasked by the reaction with gliadin [16]. The association between the intestinal increased permeability and the small intestinal bacterial overgrowth (SIBO), may reflect qualitative and quantitative changes in the microbiota, which lead to the disruption of the intestinal barrier, the subsequent bacterial translocation and the development of portal endotoxemia [17, 18]. Miele et al. [19] reported that in NAFLD patients, gut permeability and the prevalence of SIBO correlated with the severity of steatosis but not with the presence of NASH. However, the mechanisms of the intestinal permeability changes include some other factors, acetaldehyde generation from alcohol, nitric oxide production, and the alterations in individual nutrients $[12,14]$.

Wigg et al. [20] assessed SIBO in 22 patients with NASH using a combined ${ }^{14} \mathrm{C}$-D-xylose and lactulose breath test. SIBO was detected in $11(50 \%)$ patients with NASH. Subsequently, the same authors reported that NASH patients were tested for antibodies against gliadin (AGA) IgA and IgG. Three patients had positive antibodies (one positive for both antibodies, and two positive only for $\mathrm{IgG}$ ), and in one of these patients in whom it was possible to perform an upper endoscopy, the diagnosis of $\mathrm{CD}$ was histologically confirmed [21]. Grieco et al. [22] observed 30 patients with laboratory diagnosis of NASH. All patients had serum assays of AGA $\operatorname{IgG}$ and $\operatorname{IgA}$ and endomysium antibody (EMA), and duodenal biopsies were collected from those who were EMA positive. Four (13.3 \%) of these patients were thus diagnosed as having occult $\mathrm{CD}$. Four patients were placed on a gluten-free diet (GFD) and after a year, the transaminase levels were normalised, and duodenal histology was improved. Nehra et al. attempted to determine whether any relationship existed between steatohepatitis and CD by investigating the serology of 47 NASH patients with high body mass index (BMI). However, only one patient $(2.1 \%)$ was IgA EMA positive [23]. Subsequently, Bardella et al. [18] investigated 59 patients with persistent hypertransaminasemia and a BMI $>25 \mathrm{~kg} /$ $\mathrm{m}^{2}$ after at least 6 months of hypolipidic diet, by liver biopsy. NASH was detected in $38(64 \%)$ of the 59 patients whereas in 21 (36\%) histology showed only steatosis. The tissue IgA transglutaminase antibodies (anti-tTG) were positive in six $(10 \%)$ patients and IgA EMA in two $(3.4 \%)$. Only two $(3.4 \%)$, positive for both markers, resulted to have $\mathrm{CD}$ based on histological findings. After 6 months of GFD, the liver enzymes normalised in both cases. Another Italian study of 121 patients with biopsyproven NAFLD reported that the prevalence of CD found at endoscopy and confirmed by histology, with $\operatorname{IgA}$ and $\operatorname{IgG}$ anti-tTG and IgA EMA positivity, was $3.3 \%$ [24]. Recently, Rahimi et al. [25] evaluated the prevalence of CD in an Iranian small population with NAFLD. Three hundred sixteen patients defined as NAFLD, based on the elevated transaminase levels, liver ultrasound and/or liver biopsy were enrolled. CD was confirmed in seven patients (2.2\%), with positive antibodies (IgA EMA and IgA anti-tTG) and histopathologic features of $\mathrm{CD}$, significantly higher than rates reported in the general population of Iran (1/164). 
Moreover, considering only the subjects with a BMI value $<27 \mathrm{~kg} / \mathrm{m}^{2}$, the prevalence of CD was $5.8 \%(7 / 120)$.

\section{Conclusion}

A pathogenetic link between increased gut permeability, microbiota and diet, can help to understand the role of gutliver axis in the development of NAFLD. However, other factors seem to play a role. Based on the available data, it can be concluded that the serological evaluations for $\mathrm{CD}$ with anti-tTG and EMA, should be a part of the general workup in the asymptomatic patients with NAFLD when metabolic risk factors are not evident, and in the patients with steatohepatitis when other causes of the liver disease are excluded.

Conflict of interest The authors declare that they have no competing interests.

\section{References}

1. P. Angulo, Non-alcoholic fatty liver disease. N. Engl. J. Med. 346, 1221-1231 (2002)

2. S. Bellentani, F. Scaglioni, M. Marino et al., Epidemiology of non-alcoholic fatty liver disease. Dig. Dis. 28, 155-161 (2010)

3. G.A. Garinis, B. Fruci, A. Mazza et al., Metformin versus dietary treatment in nonalcoholic hepatic steatosis: a randomized study. Int. J. Obes. 34, 1255-1264 (2010)

4. P.H. Green, C. Cellier, Celiac disease. N. Engl. J. Med. 357, 1731-1743 (2007)

5. L. Maiuri, C. Ciacci, I. Ricciardelli et al., Association between innate response to gliadin and activation of pathogenic $\mathrm{T}$ cells in celiac disease. Lancet 362, 30-37 (2003)

6. A. Vilppula, K. Kaukinen, L. Luostarinen et al., Increasing prevalence and high incidence of celiac disease in elderly people: a population-based study. BMC. Gastroenterol. 9, 49 (2009)

7. U. Volta, Pathogenesis and clinical significance of liver injury in celiac disease. Clin. Rev. Allergy Immunol. 36, 62-70 (2009)

8. C.P. Day, O.F. James, Steatohepatitis: a tale of two "hits"? Gastroenterology 114, 842-845 (1998)

9. C.P. Day, Genes or environment to determine alcoholic liver disease and non-alcoholic fatty liver disease. Liver Int. 26, 1021-1028 (2006)
10. S. Prakash, L. Rodes, M. Coussa-Charley et al., Gut microbiota: next frontier in understanding human health and development of biotherapeutics. Biologics 5, 1-86 (2011)

11. J.P. Nolan, The role of intestinal endotoxin in liver injury: a long and evolving history. Hepatology 52, 1829-1835 (2010)

12. M.T. DeMeo, E.A. Mutlu, A. Keshavarzian et al., Intestinal permeation and gastrointestinal disease. J. Clin. Gastroenterol. 34, 385-396 (2002)

13. A. Farhadi, S. Gundlapalli, M. Shaikh et al., Susceptibility to gut leakiness: a possible mechanism for endotoxaemia in non-alcoholic steatohepatitis. Liver Int. 28, 1026-1033 (2008)

14. T.H. Frazier, J.K. DiBaise, C.J. McClain, Gut microbiota, intestinal permeability, obesity-induced inflammation, and liver injury. JPEN J. Parenter. Enteral. Nutr. 35(5 Suppl), 14S-20S (2011)

15. P. Brun, I. Castagliuolo, V. Di Leo et al., Increased intestinal permeability in obese mice: new evidence in the pathogenesis of nonalcoholic steatohepatitis. Am. J. Physiol. Gastrointest. Liver Physiol. 292, G518-G525 (2007)

16. L. Abenavoli, V. Arena, F. Giancotti et al., Celiac disease, primary biliary cirrhosis and helicobacter pylori infection: one link for three diseases. Int. J. Immunopathol. Pharmacol. 23, 1261-1265 (2010)

17. I.R. Korponay-Szabo, T. Halttunen, Z. Szalai et al., In vivo targeting of intestinal and extraintestinal transglutaminase 2 by coeliac autoantibodies. Gut 53, 641-648 (2004)

18. M.T. Bardella, L. Valenti, C. Pagliari et al., Searching for coeliac disease in patients with non-alcoholic fatty liver disease. Dig. Liver Dis. 36, 333-336 (2004)

19. L. Miele, V. Valenza, G. La Torre et al., Increased intestinal permeability and tight junction alterations in nonalcoholic fatty liver disease. Hepatology 49, 1877-1887 (2009)

20. A.J. Wigg, I.C. Roberts-Thomson, R.B. Dymock et al., The role of small intestinal bacterial overgrowth, intestinal permeability, endotoxaemia, and tumour necrosis factor alpha in the pathogenesis of non-alcoholic steatohepatitis. Gut 48, 206-211 (2001)

21. A.J. Wigg, A.G. Cummins, Reply to Grieco et al. Gut. 49, 596 (2001)

22. A. Grieco, L. Miele, G. Pignatoro et al., Is coeliac disease a confounding factor in the diagnosis of NASH? Gut 49, 596 (2001)

23. V. Nehra, P. Angulo, A.L. Buchman et al., Nutritional and metabolic considerations in the etiology of nonalcoholic steatohepatitis. Dig. Dis. Sci. 46, 2347-2352 (2001)

24. O. Loiacono, S. Petta, G. Venezia et al., Anti-tissue transglutaminase antibodies in patients with abnormal liver test: is it always celiac disease? Am. J. Gastroenterol. 100, 2472-2477 (2005)

25. A.R. Rahimi, N.E. Daryani, H. Ghofrani et al., The prevalence of celiac disease among patients with non-alcoholic fatty liver disease in Iran. Turk. J. Gastroenterol. 22, 300-304 (2011) 